\title{
ORDRE ET DÉSORDRE PENDANT LES ENTERREMENTS DE LA MISÉRICORDE DE BRAGA À L'ÉPOQUE MODERNE
}

\author{
POR \\ MARIA MARTA LOBO dE ARAúJO \\ Universidade do Minho - Portugal. Lab2PT \\ martalobo@ics.uminho.pt
}

\section{RÉSUMÉ}

Ce travail prétend analyser les relations entre la Miséricorde de Braga et les autres confréries de la ville, le chapitre, les églises paroissiales et le tiers-ordre tout au long de l'Époque Moderne, lors des enterrements. Dans un univers peuplé par des centaines de confréries, les enterrements étaient des occasions de désordres, entretenaient des conflits et étaient à l'origine des moments de grande tension entre les plusieurs centaines de confréries qui existaient dans la ville, parmi lesquelles ressortissait la Miséricorde qui, à l'Époque Moderne, était la confrérie la plus puissante de la ville.

MOTS CLEFS: Enterrements; désordre; ordre; Miséricorde de Braga.

\section{ORDER AND DISORDER IN THE BURIALS OF MISERICÓRDIA OF BRAGA IN THE MODERN AGE}

\section{ABSTRACT}

This work want to analyze the relationships of the Misericórdia of Braga, with regard to the burials, with the other brotherhoods, the Cabido, the parish churches and the third order of the city, along the Early Modern Age.

In a universe populated by hundreds of brotherhoods, the burials caused disorders, feeding conflicts and originated moments of great tension between different religious associations of the city, among which stands out the Misericórdia, the most powerful brotherhood of the city in the Early Modern Age.

KEY WORDS: Burials; disorder; order; Misericórdia of Braga.

\section{ORDEN Y DESORDEN EN LOS ENTIERROS DE LA MISERICORDIA DE BRAGA EN LA EDAD MODERNA}

\section{RESUMEN}

El presente trabajo pretender analizar las relaciones de la Misericordia de Braga, en lo que atañe a la celebración de los entierros, con las restantes cofradías, el cabildo catedralicio, las iglesias parroquiales y la orden tercera de la ciudad, a lo largo de la Edad Moderna.

En un universo poblado por centenares de cofradías, los entierros causaban desórdenes, alimentaban conflictos y daban origen a momentos de gran tensión entre las diferentes asociaciones religiosas de la ciudad, de entre la que destaca la Misericordia, la hermandad más poderosa de la ciudad en la Edad Moderna.

PALABRAS CLAVE: Entierros, desorden, orden, Misericordia de Braga. 


\section{INTRODUCTION}

La question des conflits à propos des enterrements ce n'est pas nouvelle, comme le revèlent des travaux des spécialistes nationaux et étrangers. Les Confreries, Les Ordres Terciaires, les Curés, les Chapitres et les Miséricordes se sont toujours enfoncés en désordres tout au long de l'Époque Moderne, parfois, de façon plus au moins prolongée, d'après les capacités des parties respectives pour résoudre la question et, ainsi, pouvoir faire revenir l'ordre établie. Les Miséricordes portugaises ont eu un rôle actif dans ces conflitualités au niveau local, belligérant à certains moments avec plusieurs institutions et personnes, à cause des enterrements et bien d'autres choses. À ces moments, surgissaient des problèmes internes, ainsi qu'avec d'auttes institutions de la communauté, donnant lieu au mal-être et aux desordres qui pouvaient se prolonger dans le temps.

D’ailleurs, les Miséricordes portugaises ont surgi dans un moment de réforme de l'assistance européenne et de profonde rénovation spirituelle. La fondation de la Miséricorde de Lisbonne, en 1498, a ouvert le chemin à la prolifération de ces institutions sur le Continent et dans l'Empire. Elles étaient soutenues par les élites et sont devenues très populaires. Créées par la couronne, elles ont toujours été bénéficiées par cette dernière, qui facilitait leur implantation et, en même temps, l'exercice des 14 œuvres de miséricorde. ${ }^{1}$ Pour les appuyer, la Maison Royale les combla de privilèges, destinés aux institutions elles-mêmes, ainsi qu'à leurs membres. Cette action de la couronne avait pour objectif de les pourvoir de mesures et de moyens dans le sens de faciliter leur appui, leur développement et leur consolidation. Parmi les divers privilèges reçus, ces confréries ont, à partir de 1593, jouit du monopole de l'utilisation du brancard. ${ }^{2}$

Probablement née en 1513, la Santa Casa da Misericódia de Braga (Sainte Maison de la Miséricorde de Braga) ${ }^{3}$ a été fondée par l'archevêque Diogo de Sousa (1505-1532) et a toujours été protégée par tous les archevêques de la ville qui y détenaient un pouvoir temporel et spirituel. La confrérie a été fondée dans une chapelle du Siège épiscopal, faite construire par cet archevêque qui avait décidé qu'elle serait

1 Abreu, L. 1999. Memórias da Alma e do Corpo: a Misericórdia de Setúbal na Modernidade. Viseu: Palimage Editores; Sá, I. G. 2001. As Misericórdias Portuguesas de D. Manuel I a Pombal. Lisboa: Livros Horizonte; Lopes, M. A. 2000. Pobreza e Controlo Social em Coimbra. 1750-1850. Vols. I, II. Viseu: Palimage Editores; Pardal, R. 2013. Práticas de caridade e assistência em Évora 1650-1750: Évora: Universidade de Évora. Dis. de Doutoramento policopiada; Magalhães. A. 2013. Práticas de caridade na Misericórdia de Viana da Foz do Lima (séculos XVI-XVIII). Viana do Castelo: Santa Casa da Misericórdia de Viana do Castelo; Ramos, M. O. N. 2013. A gestão dos bens dos mortos na Misericórdia de Arcos de Valdevez: caridade e espiritualidade (séculos XVII-XVIII). Braga: Universidade do Minho. Dis. de Doutoramento policopiada. Portugaliae Monumenta Misericordiarum.

2 Consulter à ce sujet Abreu, L. 2002. "As Misericórdias de D. Filipe I à D. João V", in J. P. Paiva (coord. scientifique), Portugaliae Monumenta Misericordiarum. vol. 1: 48 Lisbonne: Centre d'Études d'Histoire Religieuse; União das Misericórdias Portuguesas.

3 Les Miséricordes étaient des confréries laïques, qui opéraient avec des numerus clausus, et qui étaient composées par des hommes, nobles et officiers, en nombre égal. Sá, I. G. 2013. As Misericórdias portuguesas séculos XVI a XVIII. 20-22. Rio de Janeiro: Fundação Getúlio Vargas. son mausolée, ainsi que celui d'autres dignités de l'Église. En 1558, il décida de sortir de cet espace et de construire des installations propres, contiguës au Siège épiscopal.

Après avoir été consolidée et lors d'un processus de croissance, la Miséricorde de Braga a reçu, en 1559, l'administration de l'hôpital Saint-Marc. Cette institution avait été, jusque là, temporellement protégée par la municipalité et spirituellement protégée par les archevêques. Ce fut l'archevêque Bartolomeu dos Mártires (1558-1589) qui réalisa la transmission, conférant ainsi, à partir de cette époque-là, de nouvelles responsabilités à la Sainte Maison. L'hôpital possédait son propre patrimoine, dans lequel s'intégraient, au-delà de diverses propriétés, trois chapelles ou de petites églises: celle de Saint-Marc, celle du Saint Esprit, et celle de Saint-Lazare. Avec son incorporation, cette institution, en plus de son église, a commencé à gérer trois temples supplémentaires, bien que la chapelle de SaintMarc ait postérieurement disparu, ne restant que celle du Saint-Esprit puisque plus tard, la chapelle de Saint-Lazare, à Senhora-a-Branca, a fini par être vendue. ${ }^{4}$ Postérieurement, l'église du Saint-Esprit a été démolie. Une autre église a été construite à sa place, consacrée à Saint-Marc.

Les Miséricordes accomplissaient, depuis toujours, la septième œuvre de miséricorde corporelle «enterrer les morts" et la septième oeuvre de miséricorde spirituelle "prier Dieu pour le prochain et pour les morts". ${ }^{5}$ Comme il s'agissait de l'une des "principales œuvres de miséricorde", ces confréries consacraient toute leur attention à l'enterrement des morts, puisqu'elles reconnaissaient l'importance que le salut de l'âme occupait dans la vie des hommes et l'investissement fait par eux pour l'atteindre. Pour cela, elles s'entourèrent de personnes et s'équipèrent de biens, de façon à pouvoir répondre efficacement au service des enterrements.

L'investissement fait lors de la mort était le reflet des privations que les personnes supportaient lorsqu'elles étaient vivantes, du sentiment d'insécurité causé non seulement par les épidémies mais aussi par la faim, bien souvent présentes dans le quotidien des populations les moins favorisées, ainsi que par la peur. La foi déposée dans le salut renforçait le besoin d'intervention des vivants et des nombreuses célébrations que ces derniers se chargeaient de faire célébrer pour les défunts. C'était aussi une façon de maintenir, parmi les vivants, l' "espoir de la résurrection". ${ }^{6}$

Tout était nécessaire pour affronter le moment d'incertitude que la mort constituait. La peur de ne pas avoir une mort accompagnée préoccupait tous les hommes, puisque l'on comprenait que la conjugaison d'efforts, à travers les prières au moment du passage, pouvait l'adoucir. Les croyants profitaient de la préparation de ce moment pour organiser leur post mortem, investissant en œuvres de charité, en célébrations, sollicitant aux vivants de prier pour

4 Cette chapelle faisait partie du complexe de la léproserie, qui intégrait une des maisons, une cour et la chapelle en question.

Compromisso da confraria da Sancta Caza da Misericordia de Lisboa, fundada pela rainha D. Leonor deLencastre. 1929: 11: Caldas da Rainha: Pipografia Caldense.

6 Araújo, A. C. 1898. "Morte, memória e piedade baroca". Revista de História das Ideias, vol. 11: 141. 
eux dans le sens de les aider à sortir du Purgatoire. ${ }^{7}$ Dû à ce lieu intermédiaire entre le ciel et l'enfer, la mort gagna une énorme importance chez les vivants. II fallait donc la préparer avec antécédence. Comme tous savaient qu'un jour, ils mourraient, la mort pouvait être préparée avec antécédence, bien que les testaments prouvent que c'était surtout lorsque la dernière heure approchait que les décisions étaient prises sur les biens d'âme, l'enterrement et le partage de l'héritage. Avoir une bonne mort et gagner le salut représentaient un but que tout le monde voulait atteindre.

\section{LA MISÉRICORDE DE BRAGA ET LA MORT}

Pendant les XVII ${ }^{\text {ème }}$ et XVIII ème siècles, la mort atteignit une plus grande expression avec l'exagération baroque. On pouvait assister à des cérémonies grandioses et pompeuses, totalement en harmonie avec la représentation locale de qui les organisait. La Miséricorde de Braga accomplissait cette œuvre de miséricorde en allant chercher les défunts chez eux, en enterrant ceux qui décédaient à l'hôpital SaintMarc et ceux qui étaient trouvés morts dans les rues, en célébrant des messes et en chantant des répons pour leurs âmes. Pour accomplir ce service, elle possédait initialement un brancard et divers chapelains.

La possession de brancard propre doit dater de l'année 1585. Lorsque l'augmentation du nombre de frères a été débattue, il a été considéré que les diverses Miséricordes particulièrement celles de Lisbonne et de Porto, étaient pourvues de brancards et de bannières particulières qui étaient utilisés pendant les funérailles des frères et de leurs femmes. Dans ce sens, les frères de Braga décidèrent d'acquérir ce meuble, couvert de tissus de velours et de toile dorée, ainsi que la bannière ${ }^{8}$ et, l'année suivante, ils ont fait une quête parmi les frères de façon à pouvoir procéder à ces acquisitions. $^{9}$

L'engagement des frères dans l'achat de biens considérés essentiels pour l'institution pendant ses premiers temps d'existence était aussi courant dans d'autres confréries congénères. Le recours à des quêtes et à la contribution des membres du comité a eu lieu dans de nombreuses Miséricordes. En effet, au début, les «Santas Casas» recouraient fréquemment à cette forme de recueillir des fonds pour concrétiser quelques-uns de leurs projets. ${ }^{10}$

Dans les statuts, il est mentionné que, en 1628, la Sainte Maison disposait de trois brancards différents, du même nombre de bannières, et de suffisamment de cierges

7 Voir le Goff, J. 1993. O nascimento do Purgatório: 25-26. Lisbonne: Editorial Presença.

8 Nous ne savons pas comment était fait le transport des défunts. ADB, Fundo da Misericórdia, Livro de Irmãos 1585-1711, n.e 51, non paginé.

9 II a été déterminé que la quête serait réalisée par les fidèles et par deux frères. Arquivo Distrital de Braga (Archive du district de Braga, dorénavant ADB), Fundo da Misericórdia, Livro de termos ou actas 1586$1596 \mathrm{n}$ ㅇ 3, fls. 30v.-31.

10 Lire Magalhães, A. 2013. Práticas de caridade na Misericórdia de Viana da Foz do Lima (séculos XVI-XVIII). 229-233. Viana do Castelo: Santa Casa da Misericórdia de Viana do Castelo. Araújo, M. M. L. 2013. "Os prometimentos dos mesários da Misericórdia de Caminha no século XVII". Estudos Regionais, II série 7: 85-95. destinés aux accompagnements funèbres. Cette amplification dans l'acquisition d'objets funéraires était proportionnelle à la croissance de la ville et de la propre institution.

Sur l'un des brancards étaient transportés les pauvres, les souffrants et les personnes considérées "communes», alors que celles de " plus grande prestige » étaient transportées sur un autre brancard. Les frères et "d'autres personnes que la confrérie accompagnaient" étaient aussi transportés sur un brancard différent. Un plus grand nombre de brancards était prévu, en cas de besoin.

L'engagement pris convenait des procédés à adopter face à la mort d'un confrère et demandait la présence obligatoire de tous, revêtus de balandras et transportant des cierges. Cet engagement déterminait aussi en détail la composition et l'organisation du cortège funèbre. Les femmes des confrères qui demeuraient veuves et honnêtes, ainsi que leurs enfants qui étaient sous le pouvoir de la patrie jouissaient des mêmes prérogatives, lorsqu'ils étaient célibataires, n'étaient pas âgés de moins de 18 ans ni de plus de 25. À cette époque, les brancards pouvaient être transportés jusqu'aux limites de la ville. ${ }^{11}$

Pour les autres enterrements, de personnes pauvres ou d'autres personnes, la Miséricorde agissait à partir d'une liste de frères obligés, pendant les mois où ils avaient été indiqués pour cette besogne, à accompagner le brancard. Ces frères étaient choisis dans les plus divers quartiers de la ville.

Les brancards de la Miséricorde étaient transportés dans un espace établi, de façon à rendre le déplacement des confrères opérationnel. Cependant, il était considéré que si un frère décédait en dehors des limites établies, il pouvait être transporté jusqu'au «lit» de sa paroisse, sans en dépasser les limites et, en ce lieu, déposé sur le brancard de la Sainte Maison. II était aussi possible d'aller au-delà de la limite établie -à condition de ne pas dépasser une lieue- ${ }^{12}$ si un héritage, d'un montant ne dépassant pas les 100 mille réis, était laissé. Postérieurement, avec la croissance du noyau urbain, nous sommes certains que les limites établies pour le transport des brancards ont été élargies.

Le paiement de l'enterrement, qui était cher, n'était pas toujours effectué immédiatement. Ce motif a mené quelques personnes à laisser des gages à la Sainte Maison pour que le cérémonial puisse être réalisé. ${ }^{13}$ Ces gages pouvaient être rachetés plus tard, ou même laissés pour payer les dépenses effectuées.

Le prix que la Sainte Maison percevait pour chaque service funèbre a souvent varié tout au long du temps. En 1627,

11 La zone de circulation comprenait un espace qui allait "jusqu'à Nossa Senhora a Branca, jusqu'à S. Vicente, jusqu'au Cruzeiro de Luiz Alvares da Cunha, jusqu'au Padrão qui était tout près de deveza das penas, jusqu'au ponte de Guimarães \& jusqu'à Santa Justa nos pellames". Compromisso da Misericordia da cidade de Braga. 1631. 23. Braga: Francisco Fernandez de Basto.

12 Distance qui, au Portugal, mesure approximativement 5 kilomètres et demi.

13 Manuel Marçal a remis, en 1621, divers objets en or et en argent comme gage d'un enterrement effectué à un membre de sa famille. ADB, Fundo da Misericórdia, Livro 4 das intradas dos Irmãos 1621-1635, n. $=54$, fl. 6 . 
il coûtait 30 cruzados. ${ }^{14}$ En effet, le Comité d'administration de la confrérie décida de réduire le prix à cause de la pauvreté qui sévissait dans la ville. Toute la première moitié du XVII ème siècle, particulièrement pendant les décennies de 20 et 30 , ont a constitué des moments de hausse d'impôts et d'aggravement des conditions de vie des populations. ${ }^{15}$

En 1630, la conjoncture motiva une nouvelle analyse du prix, car de nombreux enterrements n'étaient pas effectués par la confrérie, précisément à cause du prix. La concurrence et le manque de revenus pour payer des montants plus élevés ont été responsables pour une nouvelle baisse et une valeur de deux mille réis a été stipulée pour les personnes extérieures à la confrérie. Tous les enfants des frères ou non: les garçons jusqu'à 14 ans et les filles jusqu'à 12 ans, étaient enterrés gratuitement. Les enfants plus âgés payaient 10 cruzados. $^{16}$

Dans de nombreuses confréries de Braga, les enfants de frères, de pères et d'autres membres de la famille ne payaient aussi que la moitié du prix établi dans chacune d'elles, à condition de demeurer dépendants du chef de famille. Cette stratégie avait pour objectif de motiver les fidèles à entrer dans ces institutions. ${ }^{17}$

La baisse du prix des enterrements dans la Sainte Maison était associée à la bataille que la Miséricorde avait engagée, à cette époque, contre la confrérie de Saint Croix, sa voisine. Cette institution se trouvait dans un processus d'autonomie et d'affirmation et causait des difficultés à la Sainte Maison, au niveau de conflictualité interne, ainsi que dans le domaine des enterrements.

Ce fut par le biais de la mort que la Sainte Maison a été bénéficiée puisqu'elle a reçu de nombreux legs, ce qui a permis le développement d'un secteur qui a occupé une place très importante pendant toute l'Époque Moderne. Les legs institués finirent par remplir la sacristie et les églises de la Sainte Maison. La Miséricorde a ressentit donc le besoin de contracter un grand nombre de chapelains qui célébraient dans ses églises, dans d'autres temples et autels de la ville, désigna des frères qui avaient pour tâche de vérifier la bonne exécution de leurs besognes et prit diverses mesures pour que les attentes de ceux qui remettaient leurs dernières volontés relatives au salut de leur âme ne fussent pas trahies.

\section{LES DÉSORDRES PROVOQUÉS PAR LES ENTERREMENTS AU NIVEAU INTERNE}

Comme nous l'avons déjà indiqué, la Miséricorde enterrait gratuitement ses frères, quelques membres de leurs familles et les pauvres. Toutes les autres personnes

\footnotetext{
14 Ancienne pièce équivalent portugais de 400 reis.

15 L'analyse de la situation portugaise pendant la première moitié du XVIII ème siècle se trouve in Oliveira, A. 1991. Poder e oposição política em Portugal 1580-1640. 161-225. Lisbonne: Difel.

16 ADB, Fundo da Misericórdia, Livro de termos ou actas 15981632, n.o 4, fl. 222v.-223.

17 À propos du prix payé pour les accompagnements dans diverses confréries de la ville, voir Ferraz, T. 2012. "Acompanhar ao outro mundo: a morte nas confrarias de Braga no século XVIII. Estudo preliminar". CEM, Cultura, Espaço e Memória. Revista do CITCEM 3:170-171.
}

pouvaient pour être accompagnées par la confrérie et déposées sur son brancard à condition de payer le prix établi.

Les funérailles des frères obéissaient à un rituel propre, qui les distinguait des autres accompagnements. Le statut dont ils jouissaient leur conférait une importance accrue, qui se matérialisait en cortèges funèbres différents. Ces funérailles étaient accompagnées par tous les frères vêtus de leurs balandras et portant les brancards. II s'agissait d'ailleurs d'une obligation imposée par l'engagement pris. Appartenir à la confrérie donnait le droit de jouir de bénéfices en vie, mais surtout à l'heure de la mort.

L'ordre de la confrérie fut quelquefois perturbé pendant ces enterrements, tout au long de l'Époque Moderne, bien qu'il s'agît d'une œuvre de miséricorde accomplie envers les frères. Les sources dont nous disposons mentionnent les absences et les amendes appliquées à ceux qui n’apparaissaient point. II faut également mentionner les accompagnements de nombreux frères, effectués par d'autres confréries et leur préférence pour être enterrés dans des églises qui n'appartenaient pas à la Sainte Maison. Toutefois, les autres enterrements furent beaucoup plus problématiques. La mort rôdant constamment, les enterrements étaient fréquents et gênaient non seulement ceux qui avaient des professions, à cause de leur constante absence, mais aussi les nobles. En périodes d'épidémies, la fréquence des enterrements augmentait et le nombre de fois que le brancard sortait se multipliait, puisqu'il devait être porté et accompagné par les frères qui avaient été choisis pour le service.

Les absences à ces cérémonies devinrent récurrentes et l'indiscipline commença à régner. L'absence répétée des frères par rapport aux accompagnements eut lieu dans toutes les Miséricordes et furent transversales aux frères nobles et aux officiers. Tous prévariquèrent, démontrèrent leur déplaisir envers la tâche d'accompagner et de transporter le brancard, manquant ainsi de respect envers l'une de leurs principales obligations. Lorsque l'absence de personnes isolées ou de groupes d'hommes était remarquée, l'ordre était rompu et le désordre s'installait.

Lorsqu'il y avait des funérailles, la Sainte Maison faisait, par les rues de la ville, sonner la cloche par un salariat, appelant les confrères qui devaient emmener le brancard chez le défunt. En cortège organisé et à l'heure prévue, ils devaient se déplacer jusqu'à la demeure du défunt et transporter le brancard jusque dans l'église. Plusieurs fois, les frères ignorèrent l'appel et n'apparurent point, faisant retarder la cérémonie ou empêchant même sa réalisation à la date prévue. Tout ceci déplaisait profondément à l'institution parce qu'elle se sentait minée à l'intérieur et cette situation causait des malaises. La Sainte Maison avait, dans les enterrements, une source sûre de recettes et surtout l'occasion de capitaliser pouvoir et prestige et, également, une manière de les obtenir. ${ }^{18}$ Faire diminuer son crédit avec des actes irréfléchis

18 Lire Marcos, M. A. 2004., "La Iglesia y beneficência en la Corona de Castilla durante la época moderna. Mitos y realidades". 100-101, in Abreu, L. (ed.), Igreja, caridade e assistência na Península Ibérica (sécs. XVI-XVIII). Lisboa: Colibri; CIDHEUS; Martín, G. A. 2005. "Religiosidad y actitudes ante la muerte en la montaña noroccidental leonesa: el concejo de Laciana en el siglo XVIII". Estudios Humanísticos. Historia. 4. 163-167. 
de la part des frères tels que, par exemple, apparaître avec l'emblème d'une autre confrérie à laquelle ils appartenaient aussi était un affront susceptible de causer désordre et de les faire expulser. Donner origine à des désordres publics, abandonner le brancard, refuser de le transporter ou même laisser tomber le défunt par terre lorsqu'ils se mêlaient à la dispute de porter le cadavre avec d'autres confréries, étaient des actes considérés très graves puisqu'ils causaient des scandales et ridiculisaient la confrérie, diminuant son crédit public. ${ }^{19}$ Le fait que ces actes étaient commis devant beaucoup de monde et qu'ils pouvaient être commentés par tous augmentait la conflictualité et la sanction pouvait être aggravée.

Les prévaricateurs étaient, postérieurement, appelés devant le comité d'administration de la confrérie où ils étaient invités à s'expliquer. Ils étaient réprimandés et ils pouvaient même être expulsés au cas où les raisons données n'étaient pas considérées valables ou en cas de récidive. Lorsque cette situation avait lieu, les liens existants entre les parties étaient brisés et, bien souvent, la confiance était rompue pour toujours. Cependant, après avoir été expulsés, de nombreux frères demandaient à être réadmis quelques années plus tard. Quelques-uns d'entre eux arrivèrent à exposer leurs raisons devant les rois. Ils étaient quelquefois réadmis, même contre la volonté des confrères.

Cependant, la conflictualité n'était pas exclusive aux enterrements. Les frères commencèrent à résister devant diverses obligations, ce qui causa aussi des désordres, aussi bien dans les processions où ils n'apparaissaient pas ou dans lesquelles ils manquaient de respect envers les normes, provoquant des scandales. Le désordre s'installa aussi pendant les XVII ème et XVIII ${ }^{\text {ème }}$ siècles lorsque, assez fréquemment, au moment des élections, éclataient des conflits, montrant l'existence de factions, de conflits d'intérêts et de manœuvres bien orchestrées au sein de l'institution.

Mais pourquoi les frères prévariquaient-ils, s'ils savaient qu'ils pouvaient être expulsés? $\mathrm{Si}$, au début, les frères obéissaient avec respect à l'obligation des accompagnements, avec le temps, ils commençaient à montrer leur gêne à porter le brancard ou à apparaître aux cérémonies. Cette position était très commune chez les nobles, lesquels démontrait du mépris pour la tâche manuelle. Quelquefois, ils envoyaient leurs servants pour les remplacer. Après avoir été expulsés, comme nous l'avons déjà dit, certains frères montraient du regret et demandaient à être réadmis. Il n'était pas rare qu'ils offraient une bonne aumône pour effacer la faute commise. Lorsqu'ils étaient de nouveau acceptés, l'ordre revenait et l'espoir que le désordre ne s'installerait plus revenait.

L'absence des frères aux enterrements était aussi sujette à des amendes. La Miséricorde tenta, au long des XVII ème et $\mathrm{XVIII}^{\mathrm{ème}}$ siècles, diverses modalités de condamnation, mais aucune ne donna de bons résultats. Bien au contraire, les fautes se renouvelaient de plus en plus, ce qui prouvait que le problème était grave. En même temps, pour attirer de

19 Araújo, M. M. L. 2009. "Les élites en conflits: les Misericórdias portugaises aux $\mathrm{XVI}^{\mathrm{e}}-\mathrm{XVIII}{ }^{\mathrm{e}}$ siècles. Semata, $\mathrm{n} . \stackrel{0}{21}$, Universidade de Santiago de Compostela: 187-199. nouveaux frères et devenir plus compétitive, la confrérie augmenta petit à petit le nombre de messes pour l'âme de chaque frère décédé. En 1744, cent messes étaient dites. Dans le sens de contraindre les frères à être présents aux funérailles, la Sainte Maison détermina, en 1732, comme punition, de soustraire tous les suffrages aux prévaricateurs. ${ }^{20}$ Cette tentative n'eut aucun succès une fois que l'âme commençait à perdre du terrain en faveur du corps. C'est en 1667 que, avec les autres membres du comité d'administration de la confrérie, ${ }^{21}$ le révérend Manuel Freire de Brito, médiateur, débatit le problème du préjudice de l'institution étant donné que cette dernière n'arrivait pas à monopoliser les enterrements de la ville, ni à projeter son reflet dans l'assistance aux pauvres. ${ }^{22}$

Mais si l'existence d'autres confréries qui opéraient dans le négoce des enterrements était certaine, il n'en était pas moins vrai que les frères de la Miséricorde montraient de plus en plus leur rénitence envers cette tâche. Ils manquaient, ils n'obéissaient pas à leurs engagements, ni aux ordres des comités, ils accompagnaient d'autres confréries dont ils étaient également membres, ils manquaient de respect publiquement envers la Sainte Maison, ou demandaient à une autre confrérie d'accompagner leurs funérailles. Cette situation devint récurrente et si, en 1694, un

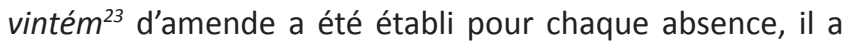
aussi été décidé que celui qui n'accomplirait pas ne serait expulsé qu'après six absences et 18 réprimandes. Si cette résolution prouve, apparemment, la volonté de réprimander les manquants, l'établissement d'un si grand nombre de fautes démontre le manque de fermeté de l'institution dans le sens de résoudre son problème et même une certaine complaisance envers les coupables.

Cette résolution a été prise après disposition du roi selon laquelle les membres du comité d'administration de la confrérie étaient autorisés à appliquer une amende à ceux qui manquaient aux accompagnements funèbres. ${ }^{24}$ Comme ce sujet n'a pas été résolu de cette façon, en 1698, la Sainte Maison, obtint du roi une permission qui autorisait à appliquer une amende de 540 réis à ceux qui manquaient aux enterrements des frères et à les expulser s'ils manquaient plus de neuf fois. ${ }^{25}$

Encore que la Sainte Maison puisse avoir le monopole des enterrements, au XVIII siècle, le nombre des confréries qui avaient son propre cercueil était très élevé, pouvant, de cette façon, entrer en concurrence avec la Sainte Maison. ${ }^{26}$ Peut-être dans le but de diminuer les absences des frères

20 ADB, Fundo da Misericórdia, Livro de termos ou actas 17231734, n. ㅇ 12, fl. 277.

21 Les Miséricorde s'étaient gérées par un comité d'administration de la confrérie, composé de 13 frères, de 7 nobles et de six officiers.

22 ADB, Fundo da Misericórdia, Livro de termos ou actas 16611678 , n. ㅇ 18, fl. 138.

23 Ancienne pièce équivalent portugais a 20 réis.

24 ADB, Fundo da Misericórdia, Livro das provizois da Caza e do Hospital, n. -704 , fl. 23.

25 Archive Nacional de la Torre do Tombo, Chancelaria de D. Pedro II, Livro n. ㅇ 17, fl. 136.

26 Lire Ferraz, Tiago. 2014. A Morte e a Salvação da Alma na Braga Setecentista. 212-216. Braga: Universidade do Minho. Dis. de Doutoramento policopiada. 
de la Miséricorde, mais aussi dans celui de rationaliser I'utilisation du brancard, il a été résolu, en 1710, pendant la médiature de l'archevêque Rodrigo de Moura Teles (17041728), que le brancard ne sortirait pas en-dehors des limites établies, comme cela avait été autorisé, contre le paiement de six mille réis. On alléguait la vulgarisation de son utilisation par les non frères qui payaient peu, permettant que de potentiels intéressés à appartenir à la confrérie ne fissent point leur demande, étant donné que, avec peu d'effort financier, ils pouvaient utiliser ses services funèbres. II s'agissait d'un mécanisme qui obligeait indirectement à s'inscrire dans la confrérie tous ceux qui désiraient leur accompagnement et leur transport sur le brancard. Outre les brancards de la Sainte Maison, I’hôpital possédait aussi les siens. En 1741, I'hôpital possédait deux brancards destinés au transport des malades pauvres qui y décédaient.

Sans arriver à résoudre le problème qui atteignait de plus en plus de proportions, en 1732, le comité d'amnistration de la confrérie et le définitoire ${ }^{27}$ pensèrent que, avec l'augmentation du nombre de messes pour l'âme les frères décédés (il y en avait 40), ils arriveraient à attirer les frères aux enterrements. Cependant, et peut-être pour savoir quels seraient les résultats, ils décidèrent aussi de retirer ce bénéfice aux manquants et d'expulser ceux qui n'apparaissaient pas aux enterrements 12 fois par an. En même temps et pour que personne n'alléguât ignorance, il fut déterminé qu'un servant afficherait la nouvelle à la contre-porte de l'église, et serait envoyé pour, de porte en porte, appeler les frères.

Il y avait divers mécanismes utilisés pour attirer un corps d'hommes qui pût dignifier le brancard et la bannière de l'institution, mais il y avait de plus en plus de difficultés et les solutions trouvées ne résolvaient pas ce problème, ${ }^{28} \mathrm{ni}$ à Braga, ni ailleurs. En 1735, face à la récidive et à l'absence de solutions pour résoudre les absences pendant les enterrements, le comité d'administration, reconnaissant le peu d'importance que les confrères donnaient à la diminution des messes pour leur âme, et débattant le paiement de l'amende, opta pour continuer l'élimination des suffrages, faisant élaborer une liste de frères qui seraient chargés de cette fonction et de maintenir le rôle du servant dans la diffusion de la nouvelle à domicile. Le doute des membres du comité d'administration de la confrérie reflète aussi leur manque d'imagination dans le sens de forcer les confrères à exécuter une tâche à laquelle ils étaient contraints, bien qu'ils la rejetassent, ne craignant nullement les conséquences. Le manque d'efficacité des mesures prises était reconnu, mais il n'y avait pas une solution pour résoudre cette affaire.

Le petit nombre de frères présent qui sont aux enterrements causait du scandale et préoccupait les dirigeants, qui

27 Organe ayant le pouvoir de consultation qui se réunissait pour émettre des opinions sur les affaires importantes.

28 Lire, entre autres, Costa, A. F. da S. 1999. A Santa Casa da Misericórdia de Guimarães 1650-1800 (caridade e assistência no meio vimaranense dos séculos XVII e XVIII. 157-158. Guimarães: Santa Casa da Misericórdia de Guimarães. Pereira, M. D. de S. 2008. Entre ricos e pobres: a actuação de Santa Casa de Ponte da Barca (1630-1800). 234235. Braga: Santa Casa da Misericórdia de Ponte da Barca. essayaient de trouver des mesures -toutes très peu efficaces-pour résoudre le problème. Si la Sainte Maison débattait, dans diverses réunions, cette situation, elle se fit aussi ressentir sur la concurrence qui sentait, de la part d'autres confréries de la ville une diminution de leurs services et de leurs revenus. Il fallait aussi compter sur le fait que de nombreux frères demandaient à d'autres confréries de les accompagner dans leur dernière demeure. Cette situation, débattue en 1694, mena les membres du comité d'administration à décider que serait payée une amende d'un vintém, par les manquants et, en 1749, que serait payée une livre de cire. Présupposant le manque de paiement, il fut déterminé, la même année que les "rebelles" ne seraient ni reçus, ni accueillis pour quelle qu'affaires que ce soit, jusqu'à respecter cette obligation. ${ }^{29}$ Ces sanctions n'atteignirent pas l'effet prétendu et il s'avéra qu'il ne s'agissait pas d'un problème localisé, mais d'un problème qui était transversal à toutes les Meséricordes déjà étudiées. ${ }^{30}$

Sans arriver à réunir les frères, les divers comités d'administration prenaient des mesures sans efficacité, réfléchissaient sur le comportement des confrères, démontraient connaître le manque d'intérêt et les motifs de ces comportements et appliquaient des solutions sans effets pratiques: les avis, les remplacements, les amendes et les menaces n'avaient aucun effet sur les prévaricateurs qui, sans complaisance, donnaient origine à des désordres dans un secteur de fondamentale importance pour l'institution. Si, d'un côté, la fonction était méprisée et si les prévaricateurs ne démontraient aucune préoccupation, ces derniers savaient, d'un autre côté, que leur réintégration était possible l'année suivante à celle du risque.

\section{LES ENTERREMENTS ET LES DÉSORDRES EXTERNES}

Découlant des fonctions qu'elle accomplissait, la Mésiricorde de Braga maintenait des relations avec toutes les institutions de la ville. Les services religieux qu'elle prodiguait, les accompagnements funèbres et le fait que ses hommes étaient membres d'autres confréries l'obligeait à interagir avec de nombreuses personnes et institutions. Les rapports établis étaient ajustés selon les principes orienteurs de leurs règles, mais ils dépassèrent de beaucoup cet encadrement. La dispute de services et de pouvoirs et les différents tempéraments des hommes brisaient souvent l'ordre et donnèrent lieu à des désordres. II est également certain que, une fois le conflit résolu, les relations se rétablissaient et la paix et l'harmonie régnaient à nouveau.

Bien que les confréries s'organisent comme des « agents de concorde idéal » dans son contexte d'insertion,

${ }^{29}$ ADB, Livro de termos ou actas 1694-1709, n. 10, fl. 13; Livro de termos ou actas $1757-1769, \mathrm{n}$ - 16, fl. 70v.

30 Consulter, entre autres, les travaux de Pereira, M. D. S. 2008. Entre ricos e pobres..., pp. 72-85; Silva, M. J. C.. 1999. A Santa Casa da Misericórdia de Montemor-o-Velho. Espaço de sociabilidade, poder e conflito (1546-1803): 123-124. Figueira da Foz: Câmara Municipal de Montemor-o-Velho. Jardim, M. D. dos S. 1996. A Santa Casa da Misericórdia do Funchal. Século xvIII. 134-135: Coimbra: Centro de Estudos de História do Atlântico; Secretaria Regional de Turismo e Cultura. 
favorisaient identiquement des agitations et des conflits. Si, d'un côté, elles organisaient des fêtes et des pèlerinages qui contribuaient pour la cohésion sociale, d'un autre côté, encourageait des moments de désordre plus su moins graves et durables, d'après le sujet visé. La mort et les enterrements en sont concernés, étant donné qui sont associés à des questions d'argent, représentation sociale, exercice du pouvoir et même des droits paroissiaux déjà emportés. Quand ces conflits comprennent confréries puissantes, comme c'était le cas de la Miséricorde de Braga, et la mort était pratiquement une présence quotidienne, les querelles surgissaient avec plusieurs institutions et des personnes réligieuses de prestige. En outre, les disputes engagées par la Sainte Maison s'inscrivent dans la confrontation sociale de l'Époque Moderne et attestent la concurrence existante entre les institutions de la ville autour des legs pieux et la prestation des services funèbres. ${ }^{31}$

La réalisation de funérailles obligeait la Miséricorde à une constante vigilance de ses pouvoirs, pour empêcher d'autres institutions à s'en emparer. C'était une tâche gigantesque si l'on tient compte que, pendant la seconde moitié du XVIII'me siècle, outre le Chapitre, le Tiers Ordre et divers couvents il y avait, à Braga, plus de sept dizaines de confréries. ${ }^{32}$ Parfois, les confrontations engagées par la Miséricorde dans quelques secteurs de l'église de la ville, s'étendaient sur plusieurs fronts en même temps, en une dispute intense pour le monopole du transport du brancard. Les luttes engagées au niveau local s'élargissaient à maintes de ses institutions, religieuses ou laïques, démontrant la conflitualité existante dans la société de l'époque. ${ }^{33}$

La Miséricorde cherchait à maintenir sa sphère d'influence en tant que confrérie la plus importante de la ville, bien que défendre "sa géographie de pouvoir" ne fut pas toujours facile, surtout lorsque les concurrents étaient nombreux et appartenaient à différentes hiérarchies. Sans vouloir céder son pouvoir d'enterrer à d'autres confréries de la ville, la Sainte Maison lutta sur plusieurs fronts et à divers niveaux, aussi bien envers les diverses confréries, qu'envers certains curés de paroisses, qu'envers des chanoines du Siège épiscopal. Elle maintenait une surveillance serrée sur ce qu'elle considérait être un droit à soi démontrant pouvoir et prestige.

Le fait d'être des confréries qui jouissaient de «protection royale immédiate", statut obtenu à Trente, pendant ces moments de plus grandes tension et de désordre, les Miséricordes cherchaient protection et rétablissement de l'ordre auprès de la Maison Royale. Maintenir le monopole du transport du brancard, qui donnait accès à différents services religieux payés, était fondamental pour les Saintes

31 Capela, J. V. 2012. "A sociedade bracarense do século XVIII. Uma sociedade conflituosa. Contributo para a compreensão do clima social do Barroco bracarense no século XVIII". 32-36, in Oliveira, Aurélio et all (coord). O Barroco em Portugal e no Brasil. Maia: ISMAI.

32 Sur le nombre de confréries de la ville, consulter, Gomes, P. A. de C. S.. 2002. Oficiais e confrades em Braga no tempo de Pombal (contributo para o estudo do movimento e organização confraternal bracarense no século XVIII). 127. Braga: Universidade do Minho,dis. de Master polycopiée.

33 Lire Sá, I. G. 2013. As Misericórdias portuguesas, séculos XVI a xVIII. 72, 75. Rio de Janeiro: Fundação Getúlio Vargas.
Maisons. Mais les enterrements signifiaient plus qu'une source de recettes. C'était par leur biais que ces confréries acquéraient de nouveaux membres et affirmaient leur pouvoir en termes locaux. ${ }^{34}$

\section{La Miséricorde et la confrérie de Saint Croix}

Les désordres provoqués par la confrérie, voisine, de Saint Croix, durèrent depuis la dernière décennie du Xvième siècle jusqu'à la moitié du siècle suivant. Parmi d'autres aspects, le conflit concernait aussi les enterrements. Fondée en 1581 et située dans l'un des autels de l'église de l'EspritSaint de la Miséricorde, la confrérie de Saint Croix cette année-là, gagna certains droits de fonctionnalité dans cette église de façon à pouvoir exécuter ses obligations mais, en 1591, elle est entrée en conflit avec la Sainte Maison. Les deux parties s'accusaient mutuellement et la Miséricorde, en empêchant Saint Croix d'entrer dans l'espace de culte, coupait à cette dernière la possibilité de concrétiser sa fonction religieuse. De nombreuses raisons, liées à la croissance et à l'affirmation de la confrérie de Saint Croix dans la ville étaient en cause, bien que ce qui préoccupait le plus les confrères de Saint Croix résidait dans l'organisation des cortèges funèbres et dans la "saisie" de ses membres au moment de la mort, bien que quelques-uns d'entre eux étaient simultanément des confrères de la Sainte Maison et de Saint Croix. Cette confrérie avait déjà son propre brancard, à l'époque où la Miséricorde reçut le sien. La confrérie de Saint Croix avait des comportements et des attitudes qui ne pouvaient pas être tolérés par la Sainte Maison, tels que: porter le brancard sur les épaules, élever la croix et sonner la cloche pendant les enterrements. Pour la Miséricorde il s'agissait d'actes trop osés, étant donné que la Sainte Maison désirait attirer vers soi ces prérogatives. ${ }^{35}$ Le désordre qui régnait entre la confrérie des Mareantes (gens de la mer) et celle de la Miséricorde de Viana da Foz do Lima, aussi par rapport à l'utilisation de ces symboles pendant les enterrements, a été présenté à maintes reprises devant le roi, étant donné que la Sainte Maison ne consentait pas que les symboles de son pouvoir fussent utilisés par une autre institution concurrente. ${ }^{36}$

La Sainte Maison de Braga se plaignait que les confrères de Saint Croix enterraient leurs frères dans l'église, qu'ils plaçaient des inscriptions sur leurs sépultures et qu'ils

34 Ces fonctions sont analysées en Magalhães A. 2011.“Os funerais nas práticas de caridade da Misericórdia de Viana da Foz do Lima (séculos XVI-XVIII)". 104-107, in Génese e organização das Misericórdias. Actas das I Jornadas de Estudo da Misericórdia dos Arcos de Valdevez. Arcos de Valdevez: Santa Casa da Misericórdia dos Arcos de Valdevez.

35 La Miséricorde de Viana da Foz do Lima maintint une dispute de plus d'un siècle, au sujet des enterrements, avec la confrérie des Mareantes de la ville, précisement parce que cette dernière utilisait le son de la cloche et la bannière pendant lés enterrements. Lire Serra M. D. P. da C. 1995. "As duas confrarias dos Mareantes de Viana da Foz do Lima no século XVı". Estudos Regionais 16: 77-78.

36 Consulter Magalhães, A. 2007. "A pompa e a inovação": a Misericórdia de Viana da Foz do Lima e os conflitos com as confrarias dos Mareantes (1523-1623)". NW Noroeste. Revista de História. Actas do Congresso Internacional de História Territórios, Culturas e Poderes. vol. II: 368. Braga: Universidade do Minho. 
faisaient excessivement sonner la cloche, causant leur ruine. Bien que la Miséricorde ne pût empêcher les frères en question d'utiliser leur église puisqu'ils y étaient installés par bulle papale, la Sainte Maison leur rendait la vie difficile, les obligeant à rester sous leur dépendance et pouvoir. Il s'agissait d'une question de hiérarchie et de pouvoir à une époque où la question des enterrements était très sensible et où les deux intitutions se trouvaient dans un processus de croissance et d'affirmation locale.

La question de l'utilisation de la cloche pendant les cortèges funèbres était importante pour donner de la visibilité à ces cérémonies. Faire ostentation de leur présence dans la ville dans des moments importants donnait du prestige et la confrérie de Saint Croix le savait bien. C'est pourquoi elle insistait et utilisait la cloche, renforçant son capital symbolique et ne respectant pas les usages de la Miséricorde. Le désordre s'est donc installé et l'affaire arriva d'abord aux oreilles de l'archevêque, puis à celles du roi. Le roi retira visibilité à la confrérie de Saint Croix, obligeant ses frères, pendant les enterrements, à porter le brancard à la main, sans cloche et à s'habiller de toges violettes et non pas noirs, puisque cette dernière couleur était attribuée à la Miséricorde. La force des symboles était dosée en faveur de la Sainte Maison ce qui retirait publiquement le rôle principal aux frères de Saint Croix.

En 1613, lorsque cette confrérie décida d'entamer le débat sur la construction d'une église a soi, la Sainte Maison posa de nombreuses entraves, car elle ne voulait pas voir diminuer le mouvement de fidèles dans l'église, ni perdre les enterrements. Après la construction d'un siège à soi, le conflit s'installa de nouveau puisque, en 1630 , la confrérie de Saint Croix, pour devenir la plus compétitive dans le négoce des enterrements, baissa le prix de ces services, ce qui rendit les frères de la Sainte Maison furieux. L'église de Saint Croix était voisine de celle de l'Esprit-Saint et sa confrérie devint l'une des plus puissantes de la ville. Elle maintint une attitude très endurcie dans le domaine des enterrements par rapport à la Miséricorde. Les deux institutions connaissaient la force et le pouvoir de la mort et cherchaient à les utiliser en bénéfice propre. Pour cette raison, les désordres se prolongèrent pendant le XVII ${ }^{\text {ème }}$ siècle, mais durèrent moins de temps. ${ }^{37}$

\section{La Miséricorde et les prêtres de la paroisse de São João de} Souto

La confrérie de Saint Croix n'était cependant pas l'unique rivale de la Sainte Maison. L'existence d'un grand nombre de confréries dans la ville, alliée au fait qu'il y en avait plusieurs qui avaient le monopole du transport du brancard et qui pouvaient effectuer les enterrements moyennant paiement,

37 Les désordres qui existaient entre la Miséricorde et la confrérie de Saint Croix sont étudiés in Araújo M. M. L. 2012. "Entre o conflito e a cooperação: as relações da Misericórdia de Braga com a confraria de Santa Cruz na Idade Moderna". 77-93. in Culto, Cultura e Caridade. Atas do Il Congresso de História da Santa Casa da Misericórdia do Porto: Porto: Santa Casa da Misericórdia do Porto. obligeait la Miséricorde à se maintenir très vigilante et à intervenir aussitôt qu'elle le jugeait opportun et pertinent.

Les rapports avec le curé de la paroisse de São João de Souto, Gaspar de Araújo, paroisse où était situé l'hôpital Saint-Marc, n'ont pas toujours été pacifiques. Il y avait, dans cette paroisse, des désordres et une grande instabilité entre la Miséricorde et le curé en question. Le conflit éclata en 1607, lorsque Gaspar de Araújo demanda de garder pour lui le montant des fosses, des offices des défunts et des enterrements. Il appuyait cette demande sur le fait qu'il avait juridiction ecclésiastique sur les chapelles de Saint-Marc et de l'Esprit-Saint, toutes les deux administrées par l'hôpital Saint-Marc, comme nous l'avons déjà mentionné. Le curé a ouvert un contentieux à la Cour d'Appel de Braga contre la Miséricorde, dans une tentative de prouver son droit sur ces espaces religieux qu'il disait posséder depuis des décennies, accusant le chapelain de l'hôpital de garder pour lui les revenus de "nombreux enterrements" et des offrandes remises par les fidèles à l'occasion des Fidèles-de-Dieu. II soutenait que lui appartenait aussi la célébration de la messe que la Sainte Maison faisait célébrer pour chaque défunt de l'hôpital et les matines, ainsi que toutes les recettes provenant d'autres services religieux. Le curé en question cherchait à démontrer l'ancienneté de son droit, contrariant les arguments utilisés par le chapelain de l'hôpital et insinuant que si les actes religieux en question étaient pratiqués par le chapelain, ils l'étaient "en cachette"étant donné qu'il ne les avait jamais autorisés. L'idée sous-jacente à cette argumentation était qu'il protégeait religieusement la chapelle et qu'il devait avoir connaissance et autoriser tous les actes qui y étaient pratiqués.

Avec la possession de l'hôpital Saint-Marc, la Miséricorde a commencé à assister spirituellement les malades et à enterrer les défunts qui y décédaient. Saint-Marc était situé dans la paroisse de São João de Souto. Ce fait était une source de discordes par rapport à la juridiction ecclésiastique de ses lieux de culte. Le chapelain de l'hôpital était obligé de célébrer pour les malades sur les autels de l'hôpital, de leur administrer les sacrements, de les aider à bien mourir, de les enterrer et de célébrer une messe pour leur âme. À partir de 1593 , il commenca à être aidé par quatre autres chapelains.

Un par un, les arguments de l'abbé de São João de Souto ont été réfutés par le chapelain de l'hôpital, qui prouva son droit d'administrer les sacrements, d'enterrer et de célébrer la messe et les matines pour les défunts de l'hôpital, ainsi que tous les autres services religieux qu'il réalisait dans les chapelles en question, en particulier les enterrements.

La même année, l'affaire a été, initialement, résolue, avec la sentence de la Cour d'Appel de la ville, en faveur du chapelain de l'hôpital. ${ }^{38}$ Cependant, l'abbé était grandement entêté, ce qui le fit appeler au tribunal de légation de Lisbonne, où le cas resta pendant jusqu'en 1613, année où la sentence, confirmant celle de la Cour d'Appel de Braga, fut prononcée. Devant cette confirmation, l'abbé de São João de Souto essaya d'appeler, en 1616, devant le SaintSiège, bien que son appellation n'ait pas été reçue par le

38 ADB, Fundo da Misericórdia, Livro do Tombo do Hospital 1715, n. .485 , fls. $19 v .-38$. 
tribunal de légation lequel, après avoir excommunié l’abbé, l'obligea à payer tous les frais inhérents au procès. ${ }^{39}$ La prise de décision en faveur de la Miséricorde était appuyée sur le statut spirituel de l'hôpital Saint-Marc. En termes spirituels, cette institution était sous la protection des archevêques. De cette façon, le curé de São João de Souto n'avait pas de juridiction sur son église.

Traduire la Miséricorde en justice, c'était lutter contre les hommes les plus puissants de la terre. Ces institutions intégraient les élites locales, les hommes les plus influents et, dans le cas de celle de Braga, non seulement les familles les plus importantes comme de nombreux chanoines et l'archevêque lui-même. Ce serait donc entrer en litige avec les plus forts. Les membres de la Miséricorde ne faisaient pas seulement partie de cette institution. Quelquesuns d'entre eux circulaient dans les sphères de pouvoir de la ville, puisqu'ils étaient conseillers municipaux, membres de la Cour d'Appel, chanoines du Siège épiscopal, proches de l'évêque pour occuper une place au Palais et membres d'autres confréries, puissantes elles-aussi, de la ville. Comme elles opéraient à base de numerus clausus, les familles les plus importantes en termes locaux cherchaient à appartenir aux Miséricordes, se rapprochant d'institutions très privilégiées et qui géraient de nombreux biens et ressources.

En 1632, le curé de São João de Souto réclama de nouveaux des droits sur les enterrements qui étaient faits dans l'église de l'Esprit-Saint et mena l'affaire devant la Cour d'Appel de la ville. La répétition fit comprendre que ces prêtres s'entêtaient à causer du désordre et à réclamer pour eux les dividendes que les enterrements offraient, bien qu'ils acceptaient, contrariés, les décisions judiciaires. Les prêtres de São João de Souto recommencèrent à perturber l'ordre, en 1787, ce qui obligea la Miséricorde à demander à la reine la confirmation de ses privilèges. À cette époque, la confrérie accusait le curé de São João de Souto de vouloir transformer l'église de la Sainte Maison en église paroissiale, statut que ces églises n'avaient jamais eu. Après avoir analysé la documentation envoyée, dans laquelle il était prouvé que les curés de São João do Souto n'avaient pas d'autorité sur les actes religieux, tels que les enterrements dans l'église de l'hôpital Saint-Marc, la reine Marie $1^{1}$ ère à la lumière des privilèges de la confrérie, émit une disposition qui bénéficiait la Sainte Maison, en finissant avec les prétensions des religieux mentionnés ci-dessus. ${ }^{40}$ La souveraine justifia que "tout se conserve dans l'état où il se trouve en faveur de la même Miséricorde recourante en faveur de ses privilèges et aussi en ce qui concerne la visitation $" .^{41}$

La tentative des divers prêtres de São João do Souto, de défendre un droit qu'ils pensaient être le leur, est significative. Si nous tenons compte de la croissance du nombre de malades de l'hôpital, surtout pendant le XVIII ème siècle, l'importance des enterrements en termes financiers devient

39 ADB, Fundo da Misericórdia, Livro do Tombo do Hospital 1715, n. -485 , fls. 38v. -50 .

40 ADB, Fundo da Misericórdia, Livro de termos ou actas 17891791 , n. 920 , fls. $46 \mathrm{v} .-47 \mathrm{v}$.

$41 \mathrm{ADB}$, Livro curioso que contem as principais novidades sucedidas no discurso de 35 annos precipiando pello de 1755 athe o de 1790, Mas. N.․ 341, fl. 590 . de plus en plus claire, bien que l'on sache que les taux de mortalité dans l'institution étaient bas. Toutefois, il faut aussi penser aux funérailles des personnes de la ville, qui cherchaient à être enterrées dans l'église de l'hôpital.

\section{La Miséricorde et le Siège épiscopal}

La Miséricorde est née dans la paroisse du siège épiscopal, là où elle avait son complexe et son église principale. Elle maintenait, pour cette raison, une relation ombilicale et juridictionnelle avec le siège épiscopal qui la faisait interagir très souvent avec cette institution. Le siège épiscopal était, et est encore, l'institution religieuse la plus emblématique de la ville. Cependant, leurs rapports n'étaient pas toujours harmonieux. En 1632, un désordre explosa entre les prêtres de la chorale du siège épiscopal et les chapelains de la Sainte Maison, étant donné que ces derniers ne permirent pas la présence, ni le chant des prêtres de la chorale pendant les offices célébrés pour l'âme du frère Agostinho de Jesus (1588-1609), pour l'âme de l'abbé Domingos Peres et pour celle de l'archidiacre de Vermoim. Les prêtres chanteurs accusant les chapelains de la Sainte Maison de ne pas les laisser participer aux célébrations, affirmaient qu'ils avaient juridiction sur l'église de la Miséricorde, étant donné qu'elle était située dans la paroisse du siège épiscopal, qu'ils participaient depuis des décennies dans ces célébrations, qu'ils s'étaient présentés dans l'intention de participer, mais qu'ils n'y avaient pas été autorisés. Ils réclamaient aussi leur paiement, pour avoir été présents et disponibles pour le service. Comme la Miséricorde ne prétendaient pas les payer et voulaient en finir avec leur présence dans l'église pendant ces occasions, le désordre s'installa parce que les prêtres chanteurs du siège épiscopal décidèrent d'instaurer un procès judiciaire qui fut résolu en 1633 , en leur faveur. ${ }^{42}$ L'historiapraphie plus récente continue de démontrer les luttes déclenchées par les chapitres des différents diocèses en faveur des droits qu'ils considéraient établis. ${ }^{43}$

La situation était très délicate pour la Miséricorde qui se sentait fléchie par la décision du tribunal ecclésiastique, bien que cette décision ne lui plût point. L'ordre fut néanmoins rétabli et la décision respectée. Cependant, pour la Sainte Maison, il s'agissait d'un moment très difficile parce qu'elle maintenait simultanément deux autres conflits: I'un avec la confrérie de Saint Croix et l'autre avec le religieux de São João de Souto. Ces deux derniers conflits duraient déjà depuis quelques années, bien qu'il y ait eu, entretemps, des phases de compréhension.

Le désordre causé par les chanteurs du siège épiscopal pendant une période de croissance de la Miséricorde démontre une certaine audace de la part de la Sainte Maison, une

42 ADB, Fundo da Misericórdia, Livro 2 das sentenças que pertencem a esta Gaveta do Coro, distribuições e multas, doc. N. 16, sans pagination.

43 Sur les conflits déclenchés par des canons Cabidos des diocèses espagnols de Calahorra et Plasensia contre religieux et laïcs puissent voir Fernández Terricabras, Ignasi. 2008. "Una tipologia de conflictos urbanos: cabildos catedralicios y obispos en la Espanã post-tridentina". In Fortea, Jose I.; Gelabert, Juan E. (eds.). Ciudades en conflicto (siglos XVI-XVIII). 109-124. Valladolid, Junta de Castilla y León; Marcial Pons. 
fois que ses chapelains agissaient sans le consentement du comité d'administration de la confrérie. Entrer en discorde avec l'institution religieuse la plus puissante de la ville, le siège épiscopal, était une matière très délicate et seulement compréhensible à la lumière des nombreuses offrandes qui arrivaient à la confrérie par le biais des enterrements. Il ne faut pas oublier que la Miséricorde est née dans le siège épiscopal et que le complexe construit pendant la moitié du Xvième siècle, qui lui conférait une grande autonomie par rapport à ce temple, lui était contigü. D’un autre côté, la Sainte Maison était, à cette époque-là, gérée par les hommes de l'église de Braga, un grand nombre d'entre eux, chanoines du siège épiscopal. II s'est donc agi d'une lutte que la Miséricorde engageait à la recherche de consolider sa place dans la Braga Moderne, démontrant pouvoir et capacité économique pour disputer son autonomie par rapport à l'institution religieuse la plus puissante de la ville.

\section{La Miséricorde et la confrérie de Saint Pierre}

La confrérie de Saint-Pierre était religieuse, bien qu'elle reçût aussi des frères laïques. Son siège était situé au siège épiscopal. Elle possédait le droit du brancard et, en 1667, la Miséricorde se sentait lésée par ce fait et regrettait qu'il en fût ainsi. La Sainte Maison affirmait que la confrérie de Saint-Pierre appliquait les revenus qu'elle tirait des enterrements pour aider les pauvres, garantissant que cette situation était unique puisqu'aucun village et qu'aucune ville du royaume ${ }^{44}$ ne faisait cela, ce qui n'était pas vrai.

À une époque de grande pauvreté dans la ville et pendant laquelle les frais de l'hôpital augmentaient, que la Sainte Maison allégua que la confrérie de Saint-Pierre appliquait les revenus des enterrements en aumônes distribuées aux pauvres représentait un argument de poids. Bien que cela ne plût point à la Miséricorde et que cette dernière ne pouvait les empêcher d'exister, il y avait des institutions religieuses qui possédaient le droit de brancard dans la ville (quelques confréries, le chapitre et le Tiers Ordre). Pour cela, elle chercha à contourner la question et à gérer cette affaire de la façon qui lui était le plus favorable. Cependant, lorsqu'il s'agissait de frères qui demandaient à être accompagnés par d'autres confréries et transportés sur leurs brancards, le désordre s'installait à nouveau. Par exemple, le frère Domingos da Costa Araújo, décéda en septembre 1739, avait décidé d'être enterré par la confrérie de Saint-Pierre de laquelle il était membre. La Sainte Maison se réunit pour analyser la situation et décida de ne pas accompagner le cortège funèbre pour considérer qu'il était «indécent" de se placer dans une situation de subalternité face à la confrérie en question. La Miséricorde rappelait sa condition de confrérie royale pour considérer un outrage le fait d'accompagner un frère séculier «conduit par d'autres frères séculiers d'une autre confrérie et non par nos propres frères et sur notre brancard". ${ }^{45}$

${ }^{44}$ ADB, Fundo da Misericórdia, Livro de termos ou actas 1661 1678 , n.o 8, fl. 138.

45 ADB, Fundo da Misericórdia, Livro de termos ou actas 17341746 , n.o 13 , fl. $196 \mathrm{v}$.
À cette occasion et comme signe d'intolérance envers de semblables situations futures, la Sainte Maison résulut de n'accompagner que des frères chanoines qui seraient conduits sur le brancard du chapitre. Tous les confrères ecclésiastiques qui choisiraient une autre confrérie pour les conduire dans leur dernière demeure seraient privés de l'accompagnement de la Miséricorde. ${ }^{46}$ En serrant ses rangs, la Sainte Maison envoyait un message très fort à ses membres: s'ils voulaient être accompagnés, ils devraient utiliser leur brancard, étant donné que l'exception n'engloberait que les chanoines. La confrérie la plus importante de la ville se sentait supérieure à toutes les autres et ne tolérait pas d'être placée dans une situation susceptible d'être critiquée, dans des moments aussi importants que ceux des enterrements.

La position de la Miséricorde irrita les confrères de SaintPierre et ces derniers décidèrent de ne pas accompagner non plus les frères qui étaient confrères de la Sainte Maison et qui demanderaient le brancard de cette dernière pour leur enterrement. Cette position était courante à l'Époque Moderne: on répondait de la même façon jusqu'à ce que l'ordre revînt. Cependant, lorsqu'en 1746, un frère de ces deux confréries laissa en testament la volonté d'être transporté sur le brancard de la confrérie de Saint-Pierre, le comité et l'assemblée de la Sainte Maison se réunirent pour réitérer la position prise auparavant. Cependant, la Miséricorde trouva un terrain d'entente en admettant qu'elle changerait de position si sa rivale acceptait d'accompagner les frères des deux institutions qui fussent chevaliers de l'Ordre du Christ. Par rapport aux autres enterrements, la Sainte Maison décida d'accompagner ceux qui étaient frères des deux institutions, ainsi que membres du chapitre, mais seulement au cas où la confrérie de Saint-Pierre procéderait de la même façon par rapport aux frères prêtres de la Sainte Maison. ${ }^{47}$

Le fait que plusieurs frères parmi ses membres étaient chanoines ou simplement prêtres, et que quelques-uns d'entre eux appartenaient simultanément à trois institutions fit comme quoi la Miséricorde condescende et cherche à s'entendre avec la confrérie de Saint-Pierre, étant donné que la pression éprouvée en certains moments était grande. La tentative d'accord n'avait pas encore été confirmée trois ans plus tard. Pour cela, lors d'un nouvel enterrement dans les conditions antérieurement mentionnées, le désordre s'installa à nouveau. L'entente entre les parties a été atteint à une date que nous ignorons mais il est vrai que, quelques années plus tard, non seulement régnait l'entente, mais aussi la coopération entre les deux institutions.

\section{CONCLUSION}

Bien que les institutions étudiées favorissent l'ordre, il n'en est pas moins vrai que, dans le quotidien, bien souvent, le désordre s'installait. Les enterrements furent une des causes qui donnèrent origine à ces désordres, bien que d'autres

46 Sur Goa, lire Sá, I. G. 1997. Quando o rico se faz pobre: Misericórdias, caridade e poder no império português 1500-1800. 167. Lisboa, Comissão Nacional para a Comemoração dos Descobrimentos portugueses.

47 ADB, Fundo da Misericórdia, Livro de termos ou actas 17461751, n.o 14, fls. 15 v. -16 . 
raisons fussent aussi présentes au moment de l'éclatement de passions et de raisons, menant la Miséricorde et d'autres institutions de Braga à entrer en conflit. La présence de la Sainte Maison dans la mort fut très importante tout au long de l'Époque Moderne et augmenta à partir du moment où elle reçut le monopole de l'utilisation du transport sur le brancard, objet que d'autres institutions religieuses de la ville étaient aussi autorisées à utiliser. L'utilisation de ce privilège et les bénéfices qu'il lui conférait, en termes de recettes mais surtout de prestige et de pouvoir, incompatibilisa la Miséricorde avec d'autres confréries concurrentes, cette dernière se maintenant très ferme dans la défense d'une prérogative qui lui ouvrait les portes des offrandes et des suffrages. C'était aussi à travers les cérémonies publiques qu'elle réalisait lors des enterrements que la Sainte Maison recrutait des frères, recevait des recettes et de nombreuses offrandes. Comme on le sait, l'importance des offrandes, pendant cette période était énorme, bien qu'elle fût aussi responsable pour l'augmentation de nombreuses obligations, quelques-unes d'entre elles ayant un caractère perpétuel. Toutefois et malgré l'accroissement des responsabilités, aucune confrérie ne méprisait les enterrements, agissant précisément dans le sens de les gagner en détriment de leurs rivales.

Les enterrements ne furent pas seulement un motif de désordre avec les institutions qui lui étaient externes. Internement aussi, ils furent un prétexte de conflits et de désordres. Peu exécuteurs de leurs devoirs, les frères s'entêtaient à prévariquer, puisqu'ils n'apparaissaient pas aux enterrements ou causaient des scandales étant donné leur comportement en ces occasions. Devant le désordre installé et blessée dans son intérieur, la Miséricorde répondait dans la même mesure. Elle recourait quelquefois au monarque ou utilisait la force, expulsant les non exécuteurs. La réintégration dans l'ordre, le fonctionnement à travers les règles et la restauration du crédit public étaient des valeurs très importantes pour la confrérie.

En outre, la concurrence installée autour des enterrements obligea la Sainte Maison à une constante vigilance dans la défense de la prérogative qui la protégeait. Elle devint aguerrie et intolérante devant la "fuite" des enterrements vers d'autres confréries et dans la célébration de suffrages qu'elle commémorait pour les morts. La question n'était pas seulement monétaire. II y avait en cause beaucoup plus que de l'argent. ${ }^{48}$ La Sainte Maison cherchait à maintenir l'ordre internement, à solidifier sa prépondérance en termes locaux, ne permettant pas que son pouvoir fut émoussé par ses concurrentes. La défense de ce privilège entraîna avec soi des problèmes avec les paroisses, qui luttaient pour le maintien de leurs droits paroissiaux dans les espaces religieux de la Sainte Maison. Une fois la question dépassée, l'ordre s'installait à nouveau bien que cet ordre fût, dans certains cas, mis en cause par la suite, surtout lorsque revenait la question de la position de chaque institution

48 Les aspects économiques fréquemment causeurs de conflits. Lire Mantecón Movellán, Tomás. A. 1997. Conflitualidad y disciplinamiento rural del Antiguo Régime. Santander: Universidad de Cantabria; Fundación Marcelino Botín. devant la mort, les enterrements et les suffrages. Territoire d'importance capitale à l'Époque Moderne, la mort et les services qui l'entouraient furent facteurs de nombreux désordres, menant les institutions qui travaillaient dans ce domaine à s'impliquer dans la défense de leurs droits et privilèges lesquels se heurtaient bien souvent contre ceux de leurs concurrentes.

\section{SOURCES MANUSCRITES}

\section{Archive Départemental de Braga:}

Livro curioso que contem as principais novidades sucedidas no discurso de 35 annos precipiando pello de 1755 athe o de 1790, Mas. N. 341.

Livro 2 das sentenças que pertencem a esta Gaveta do Coro, distribuições e multas, doc. N. 16.

\section{Fond bibliographique de la Miséricorde :}

Livro de termos ou actas 1598-1632, n.o 4.

Livro de termos ou actas 1661-1678, n.ㅇ 8 .

Livro de termos ou actas 1694-1709, n. 10.

Livro de termos ou actas 1723-1734, n. 12.

Livro de termos ou actas 1734-1746, n. 13.

Livro de termos ou actas 1746-1751, n. 14.

Livro de termos ou actas 1757-1769, no 16.

Livro de termos ou actas 1661-1678, n.o 18.

Livro de termos ou actas 1789-1791, n. 20.

Livro de Irmãos 1585-1711, n.으. 51.

Livro 4 das intradas dos Irmãos 1621-1635, n. 54.

Livro do Tombo do Hospital 1715, n. 485.

Livro das provizois da Caza e do Hospital, n.o 704.

Archive National de la Tour du Tombo:

Chancelaria de D. Pedro II, Livro n.o 17.

Sources Imprimées:

Compromisso da Misericordia da cidade de Braga 1631. Braga: Francisco Fernandez de Basto.

Compromisso da confraria da Sancta Caza da Misericordia de Lisboa, fundada pela rainha D. Leonor de Lencastre. 1929. Caldas da Rainha: Pipografia Caldense.

\section{BIBLIOGRAPHIE:}

Abreu, Laurinda. 1999. Memórias da Alma e do Corpo: a Misericódia de Setúbal na Modernidade. Viseu: Palimage Editores.

Abreu, Laurinda. 2002. "As Misericórdias de D. Filipe I à D. João V": in J. P. Paiva (coord. scientifique), Portugaliae Monumenta Misericordiarum vol. 1: 45-67. Lisbonne: Centre d'Études d'Histoire Religieuse; União das Misericórdias Portuguesas.

Araújo, Ana Cristina. 1989. "Morte, memória e piedade baroca". Revista de História das Ideias, vol. 11: 139-152. 
Araújo, Maria Marta Lobo de. 2009. "Les élites en conflits: les Misericórdias portugaises aux XVI ${ }^{\mathrm{e}} \mathrm{XVIII}$ e siècles". Semata, n.ㅇ 21: 187-199.

Araújo, Maria Marta Lobo de. 2012. “Entre o conflito e a cooperação: as relações da Misericórdia de Braga com a confraria de Santa Cruz na Idade Moderna", in Culto, Cultura e Caridade. Atas do II Congresso de História da Santa Casa da Misericórdia do Porto: 77-93. Porto: Santa Casa da Misericórdia do Porto.

Araújo, Maria Marta Lobo de. 2013. "Os prometimentos dos mesários da Misericórdia de Caminha no século XVII". Estudos Regionais, II série 7: 85-95.

Capela, José Viriato. 2012. “A sociedade bracarense do século XVIII. Uma sociedade conflituosa. Contributo para a compreensão do clima social do Barroco bracarense no século XVIII". in Oliveira, Aurélio, Varanda, João ; Peixoto, José C.; Gonçalves, Eduardo; Pereira, Varico (coord). O Barroco em Portugal e no Brasil. Maia: ISMAI.

Costa, Américo Fernando da Silva. 1999. A Santa Casa da Misericórdia de Guimarães 1650-1800 (caridade e assistência no meio vimaranense dos séculos XVII e XVIII. Guimarães: Santa Casa da Misericórdia de Guimarães.

Fernández Terricabras, Ignasi. 2008. “Una tipologia de conflictos urbanos: cabildos catedralicios y obispos en la Espanã post-tridentina". In Fortea, Jose I.; Gelabert, Juan E. (eds.). Ciudades en conflicto (siglos XVI-XVIII). Valladolid, Junta de Castilla y León; Marcial Pons.

Ferraz, Tiago. 2012. "Acompanhar ao outro mundo: a morte nas confrarias de Braga no século XVIII. Estudo preliminar". CEM, Cultura, Espaço e Memória. Revista do CITCEM 3: 163-17.

Ferraz, Tiago. 2014. A Morte e a Salvação da Alma na Braga Setecentista. Braga: Universidade do Minho.

Gomes, Paula Alexandra de Carvalho Sobral. 2002. Oficiais e confrades em Braga no tempo de Pombal (contributo para o estudo do movimento e organização confraternal bracarense no século XVIII). Braga: Universidade do Minho, dis. de Master polycopiée.

Jardim, Maria Dina dos Santos. 1996. A Santa Casa da Misericórdia do Funchal. Século XVIII. Coimbra: Centro de Estudos de História do Atlântico; Secretaria Regional de Turismo e Cultura.

Le Goff, Jacques. 1993. O nascimento do Purgatório. Lisbonne: Editorial Presença.

Lopes, Maria Antónia. 2000. Pobreza e Controlo Social em Coimbra. 1750-1850. Vols. I, II. Viseu: Palimage Editores.

Magalhães, António. 2007. "A pompa e a inovação": a Misericórdia de Viana da Foz do Lima e os conflitos com as confrarias dos Mareantes (1523-1623)". NW Noroeste. Revista de História. Actas do Congresso Internacional de História Territórios, Cultultura. vol. II: 359-375.

Magalhães, António. 2011.“Os funerais nas práticas de caridade da Misericórdia de Viana da Foz do Lima (séculos
XVI-XVIII)", in Génese e organização das Misericórdias. Actas das I Jornadas de Estudo da Misericórdia dos Arcos de Valdevez: 99-107. Arcos de Valdevez: Santa Casa da Misericórdia dos Arcos de Valdevez.

Magalhães, António. 2013. Práticas de caridade na Misericórdia de Viana da Foz do Lima (séculos XVI-XVIII). Viana do Casatelo: Santa Casa da Misericórdia de Viana do Castelo.

Mantecón, Movellán, Tomás Antonio. 1990. Contrarreforma y religiosidad popular en Cantabria. 106107, 116-123. Santander: Universidad de Cantabria; Asamblea Regional de Cantabria.

Mantecón Movellán, Tomás Antonio. 1997. Conflictividad y disciplinamiento rural del Antiguo Régimen, Santander: Universidad de Cantábria, Fundación Marcelino Botín.

Marcos, Martín Alberto. 2004., "La Iglesia y beneficência en la Corona de Castilla durante la época moderna. Mitos y realidades ", in Abreu, Laurinda (ed.), Igreja, caridade e assistência na Península Ibérica (sécs. XVI-XVIII). Lisboa: Colibri; CIDHEUS.

Martín, García Alfredo. 2005. “Religiosidad y actitudes ante la muerte en la montaña noroccidental leonesa: el concejo de Laciana en el siglo XVIII ». Estudios Humanísticos. Historia. 4.

Pardal, Rute. 2013. Práticas de caridade e assistência em Évora 1650-1750: Évora: Universidade de Évora. Dis. de Doutoramento policopiada.

Pereira, Maria das Dores de Sousa. 2008. Entre ricos e pobres: a actuação de Santa Casa de Ponte da Barca (16301800). Braga: Santa Casa da Misericórdia de Ponte da Barca.

Oliveira, António. 1991. Poder e oposição política em Portugal 1580-1640. Lisbonne: Difel.

Ramos, Maria Odete Neto. 2013. A gestão dos bens dos mortos na Misericórdia de Arcos de Valdevez: caridade $e$ espiritualidade (séculos XVII-XVIII). Braga: Universidade do Minho. Dis. de Doutoramento policopiada.

Sá, Isabel dos Guimarães. 1997. Quando o rico se faz pobre: Misericórdias, caridade e poder no império português 1500-1800. Lisboa, Comissão Nacional para a Comemoração dos Descobrimentos portugueses.

Sá, Isabel dos Guimarães. 2001. As Misericórdias Portuguesas de D. Manuel I a Pombal. Lisboa: Livros Horizonte.

Sá, Isabel dos Guimarães. 2013. As Misericórdias portuguesas, séculos XVI a XVIII. Rio de Janeiro: Fundação Getúlio Vargas.

Serra, Manuel D. P. da Cunha. 1995. "As duas confrarias dos Mareantes de Viana da Foz do Lima no século XVI. Estudos Regionais n. 16: 77-78.

Silva, Mário José Costa da. 1999. A Santa Casa da Misericórdia de Montemor-o-Velho. Espaço de sociabilidade, poder e conflito (1546-1803). Figueira da Foz: Câmara Municipal de Montemor-o-Velho. 